T. Srinivasan-Rao and J. Smedley

Brookhaven National Laboratory

Upton, NY 11973-5000

and

K. Batchelor, J. P. Farrell, and G. Dudnikova

Brookhaven Technology Group, Inc.

25 E. Loop Road, Stony Brook, NY 11970

RECEIVED

NOV 051998

OSTI

June, 1998

DISTRIBUTION OF THIS DOCUMENT IS UNRMITED

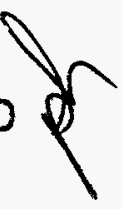

MASTER

"Work partially supported by the U.S. Department of Energy: Contract No. DE-AC02-98CH10886. 


\section{DISCLAIMER}

This report was prepared as an account of work sponsored by an agency of the United States Government. Neither the United States Government nor any ageacy thereof, nor any of their employees, makes any warranty, express or implied, or assumes any legal liability or responsibility for the accuracy, completeness, or usefulness of any information, apparatus, product, or process disclosed, or represents that its use would not infringe privately owned rights. Reference herein to any specific commercial product, process, or service by trade name, trademark, manufacturer, or otherwise does not necessarily constitute or imply its endorsement, recommendation, or favoring by the United States Government or any agency thereof. The views and opinions of authors expressed herein do not necessarily state or reflect those of the United States Government or any agency thereof. 


\section{DISCLAIMER}

Portions of this document may be illegible in electronic image products. Images are produced from the best available original document. 


\title{
Comparison of Electrostatic and Time Dependent Simulation Codes for Modeling a Pulsed Power Gun
}

\author{
T. Srinivasan-Rao,J. Smedley \\ Brookhaven National Laboratory, Upton, NY 11973 \\ K. Batchelor, J.P. Farrell, and G. Dudnikova \\ Brookhaven Technology Group Inc., 25 E. Loop Rd, Stony Brook, NY 11970
}

\section{Description of Problem}

This paper is a result of a group of simulations used to determine the optimal parameters for a pulsed power electron gun. As electrostatic codes such as PBGUNS tend to be cheaper, easier to use, and have less stringent computational requirements than time dependent codes such as MAFIA, it was desirable to determine those regimes in which the electrostatic codes agree with time dependent models. It was also necessary to identify those problems that required time dependence, such as longitudinal variation in an electron bunch. PBGUNS was then used to perform the bulk of the optimization, with only those issues that required time dependence being resolved with MAFIA.

The pulsed power gun has been discussed elsewhere [1] and consists of a diode with a flat cathode and a flat anode mounted parallel to one another with an interelectrode spacing of $1 \mathrm{~mm}$. The anode had a $.5 \mathrm{~mm}$ radius hole allowing the bunch to escape the accelerating gap. The cathode is biased at $-1 \mathrm{MV}$, yielding an accelerating gradient of $1 \mathrm{GV} / \mathrm{m}$ within the gap. The anode was $1.5 \mathrm{~mm}$ thick and was modeled as a perfect conductor and a perfect particle dump. For the purposes of simulation, the gun was assumed to be cylindrically symmetric. Figure 1 shows the simulated geometry of the gun in MAFIA, along with field lines and an electron bunch from a typical run. Figure 2 shows the geometry as it was used in PBGUNS, along with the equal potentials and particle trajectories from a typical run. Note that in MAFIA, the $\mathrm{z}=0$ boundary is used as the cathode, and the potential of $-1 \mathrm{MV}$ is set on that boundary, while in PBGUNS the cathode surface is at $\mathrm{z}=2.25 \mathrm{~mm}$. The beam parameters used for comparison were extracted as close as possible to a plane $2.25 \mathrm{~mm}$ from the inner surface of the anode. This plane was chosen so that the particles could be taken to be in the drift region, away from any fringe fields from the accelerating gap. All of the simulations modeled emission from a $.25 \mathrm{~mm}$ radius spot, but a variety of currents were modeled within that spot, all with uniform current density.

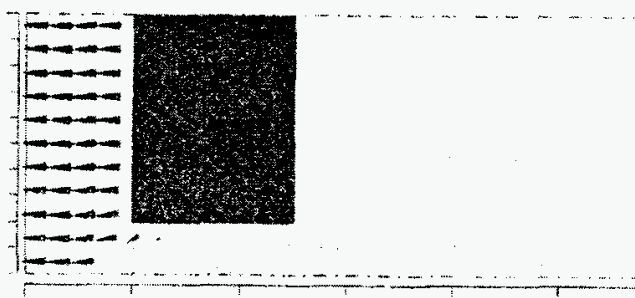

Fig 1 - MAFIA Geometry, field lines and electron bunch

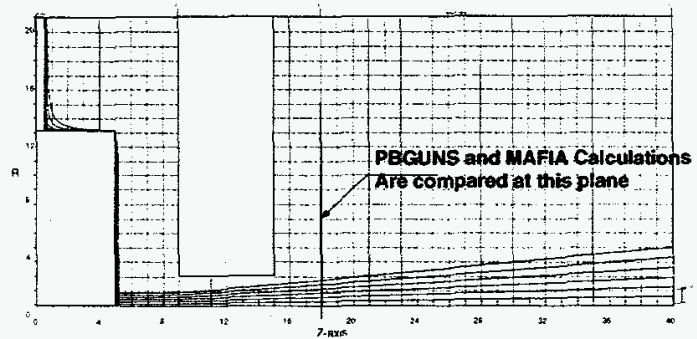

Fig 2 - PBGUNS Geometry and particle trajectories 
MAFIA is a software package that includes many electromagnetic simulation codes, including both time and frequency domain solvers in both $2.5 \mathrm{D}$ and $3 \mathrm{D}$. For this problem, the mesh generator (M) was used to set up the geometry and mesh. For all of the runs discussed below, a mesh dimension of $10 \mu \mathrm{m} \times 10 \mu \mathrm{m}$ was used. The static solver (S) was used to model the field present in the gun during the emission. The static solver solves Maxwell's equations for a given geometry and set of time- independent boundary conditions. This involves the implicit assumption that the applied field in the gun is constant during the electron bunch duration, which is valid for the device being modeled. The field generated in this manner was read into the 2D time domain particle pusher (TS2). The particle pusher handles ejection and propagation of the electrons inside the gun. It is here that the bunch duration is defined. A duration of 10 ps was used throughout the comparison, although the effect of using shorter bunch lengths was investigated. The simulation is broken into finite time steps (on the order of 10 fs for these simulations). At each time step the particles are moved under the influence of the last calculated field, while at each half time step the fields are updated due to the presence of the particles. The program combines groups of electrons into macro particles to reduce the computational resources required. Typically 75000 macro particles were used in these simulations, although fewer were used to model the shorter pulse durations. The post processor $(\mathrm{P})$ was used to extract the particle positions and momenta, both longitudinal and transverse, at the plane of interest. The comparisons were made with particles taken from the center of the MAFIA bunch, except where otherwise noted.

PBGUNS [2] is a PC compatible, 2-D code that solves Poisson's equation using iterative relaxation technique on a rectangular array of squares. It computes trajectories of charged particles in electrostatic and magnetostatic focusing systems including the effects of space charge and self-magnetic fields. Either rectangular or cylindrically symmetric geometry may be used. The Poisson equation is solved by an alternate column relaxation technique known as the semi-iterative Chebyshev method. The transverse phase space plot was extracted at the measurement plane.

\section{Bunch Length Comparison}

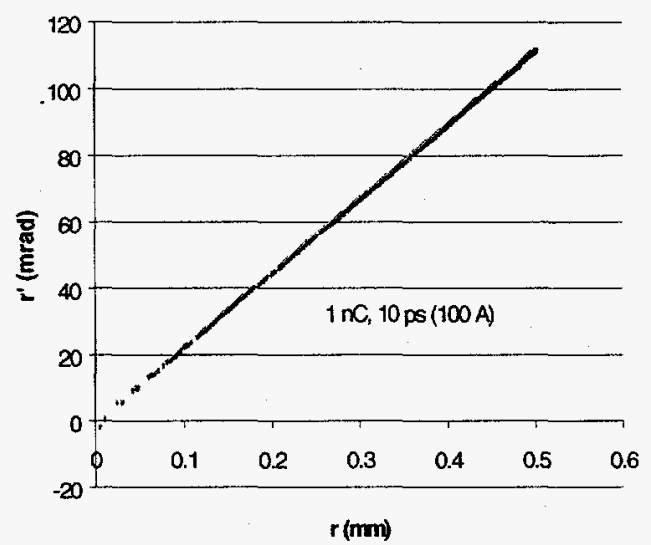

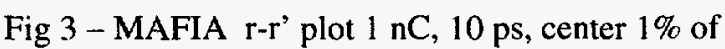
beam

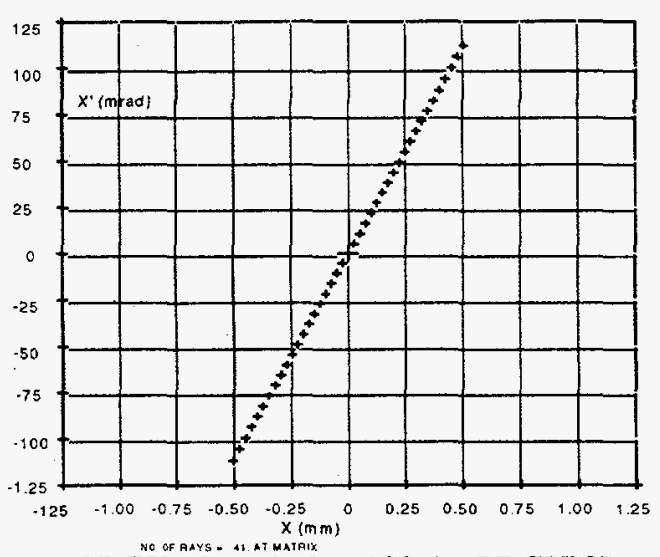

Fig 4 - PBGUNS $x-x$ p plot, 100 A. PBGUNS output comes as $\mathrm{X}-\mathrm{x}$, but for the cylindrically symmetric case this is equlivent to $r-r$ '

The first issue in the comparison of the simulation packages was "Where do MAFIA and PBGUNS agree?" In comparing the results of the simulations, a current of $100 \mathrm{~A}$ was used, with a uniform current density emission from the $.25 \mathrm{~mm}$ radius emitting spot. Fig 3 shows transverse phase space predicted by MAFIA for the center $1 \%$ of a $1 \mathrm{nC}, 10 \mathrm{ps}$ bunch accelerated in a field of $1 \mathrm{GV} / \mathrm{m}$ across the gap of $1 \mathrm{~mm}$. Fig 4 shows the DC transverse phase 
space predicted by PBGUNS for identical conditions. The transverse phase space does not vary significantly for bunch durations of $3 \mathrm{ps}, 1 \mathrm{ps}$, and $300 \mathrm{fs}$. The agreement between PBGUNS and MAFIA is very good, even for bunches much shorter than the gap transit time of 3 ps.

\section{High Charge Comparison}

The treatment of space charge effects in high current cases was investigated for MAFIA and PBGUNS. Table 1 gives the predicted maximum spot size, maximum divergence and 1- $\sigma$ slice emittance (using the center $1 \%$ of the beam for the emittance calculation) for MAFIA and the maximum spot size and divergence for PBGUNS for various currents. The method used by PBGUNS to calculate the emittance is still under investigation. For MAFIA, a bunch length of $10 \mathrm{ps}$ was used. As shown on the table, good agreement was found between MAFIA and PBGUNS for the beam radius and the max divergence. A current of 1000A is not possible in PBGUNS, as it is above the predicted DC Child's Law limit for this gun. The MAFIA results for a beam of $10 \mathrm{nC}$ (corresponding to $1000 \mathrm{~A}$ in $10 \mathrm{ps}$ ) show significant bunch lengthening in the gap. Thus while the beam is $1000 \mathrm{~A}$ initially, by the time it leaves the gun it has increased in pulse duration to about $13 \mathrm{ps,} \mathrm{thus} \mathrm{reducing} \mathrm{the} \mathrm{effective}$ current to $\sim 750 \mathrm{~A}$.

\begin{tabular}{|r|r|r|r|r|r|}
\hline & \multicolumn{2}{|c|}{ PBGUNS } & \multicolumn{3}{c|}{ MAFIA } \\
\hline CATHODE & BEAM & MAX & BEAM & MAX & NORM. \\
\hline CURRENT & RADIUS & DIVERG. & RADIUS & DIVERG. & EMITTANCE \\
\hline Ampere & mm & mrad & mm & mrad & $\pi$ mm-mrad \\
\hline 1 & 0.47 & 100 & 0.475 & 99 & 0.118 \\
\hline 100 & 0.5 & 112 & 0.503 & 112 & 0.162 \\
\hline 200 & 0.535 & 125 & 0.533 & 126 & 0.241 \\
\hline 300 & 0.6 & 140 & 0.577 & 141 & 0.292 \\
\hline 600 & 0.65 & 165 & 0.633 & 170 & 0.617 \\
\hline$\left.{ }^{*}\right) 1000$ & & & 0.707 & 173 & 2.16 \\
\hline
\end{tabular}

Table 1 - Max Spot Size, Divergence \& Emittance for 1A, 100A, 200A, 300A, 600A, 1000A for PBGUNS \& MAFIA. $\left(^{*}\right)$ The 1000 A case is above the DC limit of Child's Law for the gun. The bunch length begins stretching immediately after emission, so that the effective current is only $\sim 750 \mathrm{~A}$.

\section{Longitudinal Phase Space \& Front/Back Variation}

One aspect where time resolution is clearly required is the study of the longitudinal variation of the electron beam, both in terms of energy and transverse phase space. Fig 5 shows the longitudinal momentum spread across the beam when the center of the beam is at the measurement plane for a $10 \mathrm{ps}, 1 \mathrm{nC}$ bunch. It is interesting to note that this shape is independent of charge - the absolute extent of the front/back variation is charge dependent, but the shape is not. Fig 6 shows the longitudinal momentum spread for a $10 \mathrm{ps,} 3 \mathrm{nC}$ bunch.
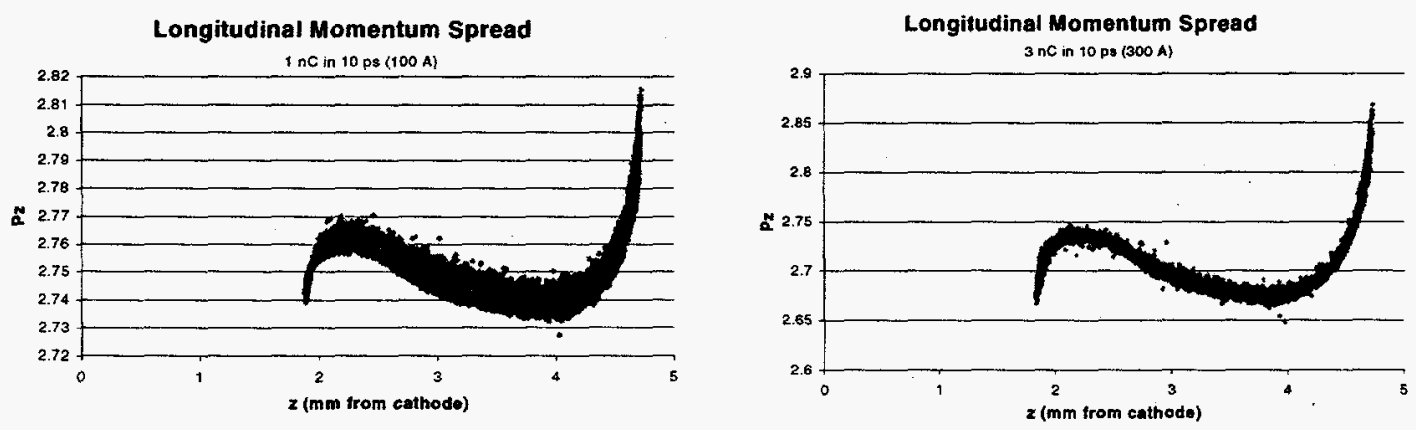

Fig 5 \& 6 Charge induced longitudinal momentum spread. $\mathrm{Pz}$ is in $\beta_{2} \gamma$. 
It is important to note that a DC code effectively measures only the slice phase space (since there is no long. variation in the beam). Fig 7 shows the MAFIA predictions for the transverse phase of slices taken from the front, middle and back of the bunch (relative to the cathode). The variation from front to back is small. It is important, however, since most diagnostic techniques measure only the integrated phase space, which would involve drawing an ellipse, which encompassed all three slices. The emittance calculated from this ellipse would be much larger than the emittance of the individual slices.

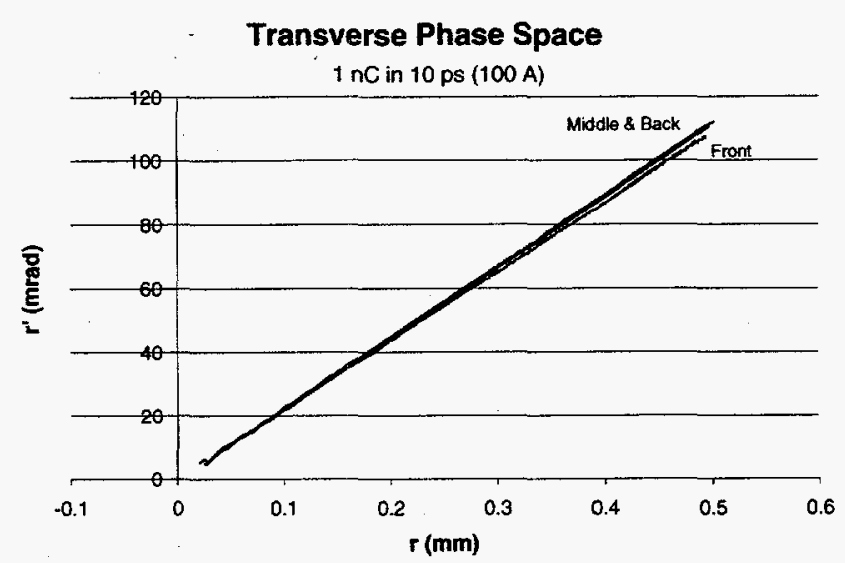

Fig 7 Longitudinal variation of the transverse phase space.

\section{Conclusion}

A comparison of two codes suitable for electron gun simulation was performed. Good agreement in transverse phase space values was found between the electrostatic code (PBGUNS) and the time dependent code (MAFIA) for a variety of pulse durations, even for pulse durations short compared to the electron transit time of the accelerating region. To obtain values for the longitudinal energy spread and the variation of the transverse phase space across the bunch, it was necessary to use MAFIA. The electrostatic codes have an advantage in terms of required computational resources and run time, and are therefor a good choice for modeling jobs in which the longitudinal energy spread is unimportant.

The authors would like to thank Harold Kirk for his assistance in utilizing MAFIA and Vadim Dudnikov for his assistance with PBGUNS.

\section{Reference:}

1. T. Srinivasan-Rao, J. Smedley, Advanced Accelerator Concepts workshop, AIP Conference Proceedings 398, Ed. S. Chattopadhyay, J. McCullough and P. Dahl, AIP Press, NY 1997, P. 7307.

2. J.E. Boers, Proceedings of the 1995 PAC, Dallas TX, pp. 2312-3, May 1995 